\title{
PTV margin definition in hypofractionated IGRT of localized prostate cancer using cone beam CT and orthogonal image pairs with fiducial markers
}

\author{
Christoph Oehler ${ }^{1,2 \dagger}$, Stephanie Lang ${ }^{1 \dagger}$, Peter Dimmerling ${ }^{1}$, Christian Bolesch ${ }^{1}$, Stephan Kloeck , Alessandra Tini ${ }^{1}$,
} Christoph Glanzmann ${ }^{1}$, Yousef Najafi ${ }^{1}$, Gabriela Studer ${ }^{1}$ and Daniel R Zwahlen ${ }^{1,2^{*}}$

\begin{abstract}
Purpose: To evaluate PTV margins for hypofractionated IGRT of prostate comparing kV/kV imaging or CBCT.

Patients and methods: Between 2009 and 2012, 20 patients with low- (LR), intermediate- (IR) and high-risk (HR) prostate cancer were treated with VMAT in supine position with fiducial markers (FM), endorectal balloon (ERB) and full bladder. CBCT's and kV/kV imaging were performed before and additional CBCT's after treatment assessing intra-fraction motion. $C T V_{P}$ for 5 patients with $L R$ and $C T V_{\text {PSV }}$ for 5 patients with IR/HR prostate cancer were contoured independently by 3 radiation oncologists using MRI. The van Hark formula (PTV margin $=2.5 \Sigma+0.7 \sigma$ ) was applied to calculate PTV margins of prostate/seminal vesicles (P/PSV) using CBCT or FM.

Results: 172 and 52 CBCTs before and after RT and $507 \mathrm{kV} / \mathrm{kV}$ images before RT were analysed. Differences between FM in CBCT or in planar $\mathrm{kV}$ image pairs were below $1 \mathrm{~mm}$. Accounting for both random and systematic uncertainties anisotropic PTV margins were 5-8 mm for P (LR) and 6-11 mm for PSV (IR/HR). Random uncertainties like intra-fraction and inter-fraction (setup) uncertainties were of similar magnitude (0.9-1.4 mm). Largest uncertainty was introduced by CTV delineation (LR: 1-2 mm, IR/HR: 1.6-3.5 mm). Patient positioning using bone matching or ERB-matching resulted in larger PTV margins.

Conclusions: For IGRT CBCT or kV/kV-image pairs with FM are interchangeable in respect of accuracy. Especially for hypofractionated RT, PTV margins can be kept in the range of $5 \mathrm{~mm}$ or below if stringent daily IGRT, ideally including prostate tracking, is applied. MR-based CTV delineation optimization is recommended.
\end{abstract}

Keywords: Prostate cancer, Radiation therapy, IGRT, VMAT, Cone beam CT, PTV margin definition, Hypofractionation

\section{Background}

Image-guided radiation therapy (IGRT) is the preferred method for curative treatment of localized prostate cancer and is associated with improved outcome and reduced toxicity [1]. IGRT using cone-beam computed tomography $(\mathrm{CBCT})$ or kilovoltage $(\mathrm{kV} / \mathrm{kV})$ gold fiducial marker (FM) imaging in combination with conformal radiation therapy (RT) techniques such as intensitymodulated radiotherapy (IMRT) or volumetric modulated

\footnotetext{
* Correspondence: daniel.zwahlen@gmx.ch

${ }^{\dagger}$ Equal contributors

'Department of Radiation Oncology, University Hospital Zurich, Zurich, Switzerland

${ }^{2}$ Department of Radiation Oncology, Hospital Graubuenden, Chur, Switzerland
}

(c) 2014 Oehler et al.; licensee BioMed Central Ltd. This is an Open Access article distributed under the terms of the Creative Commons Attribution License (http://creativecommons.org/licenses/by/2.0), which permits unrestricted use, distribution, and reproduction in any medium, provided the original work is properly credited. The Creative Commons Public Domain Dedication waiver (http://creativecommons.org/publicdomain/zero/1.0/) applies to the data made available in this article, unless otherwise stated.

arc therapy (VMAT) potentially allow smaller treatment margins and to escalate dose to the prostate [2].

PTV margins have been reduced from $10 \mathrm{~mm}$ in the pre-IGRT-era to $3-5 \mathrm{~mm}$ in the IGRT-era $[3,4]$. There is little consensus on the magnitude of PTV margin for RT of the prostate mainly due to inter-institutional technical issues and the preferred IGRT modality [5].

To compute the magnitude of a PTV-margin that allows the CTV receive the prescribed dose with a clinically acceptable and specified probability, statistics of all uncertainties in the treatment process chain should be known [6,7]. Geometrical uncertainties in RT include both treatment preparation variations and execution uncertainties. They both can be systematic such 


\section{Intersection of contours}

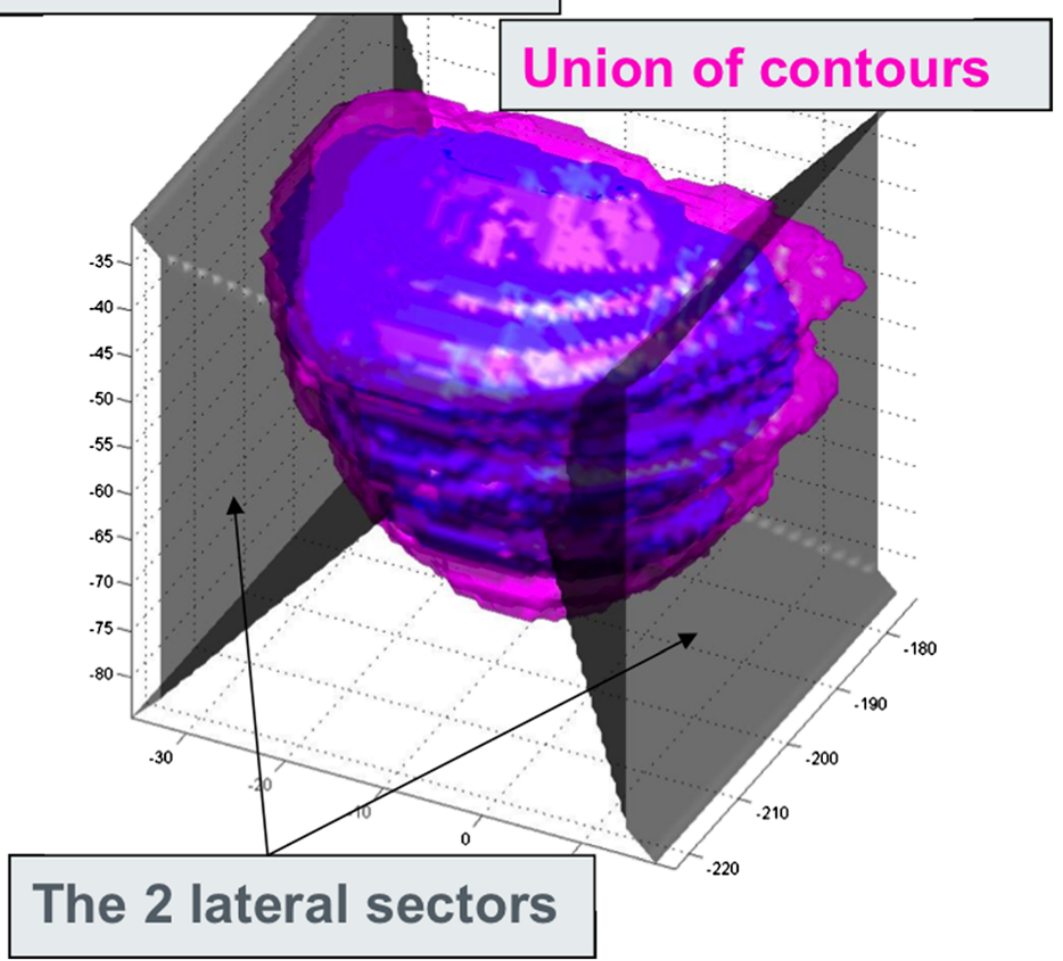

Figure 1 Depiction of the mean differences in vertical, lateral and longitudinal directions between the intersections (blue) and the unions (pink) of the three CTVp as contoured by three radiation oncologists. The prostate volume was divided into 6 subsections (anterior, posterior, left, right, cranial, caudal).

as equipment maladjustments, planning setup uncertainties, and target volume delineation or random such as treatment setup uncertainties, inter- and intra-fraction organ motion [7].

The aim of this work was to investigate PTV margin determination in the IGRT-era with endorectal balloon (ERB) calculating systematic and random treatment

Table 1 Systematic $\Sigma$ and random $\sigma$ setup error depending on surrogate used for matching $\mathrm{kV} / \mathrm{kV}$ images to the reference $C T$

\begin{tabular}{llll}
\hline & & $\boldsymbol{\Sigma}(\mathbf{m m})$ & $\boldsymbol{\sigma}(\mathbf{m m})$ \\
\hline Bones & vrt & 1.78 & 1.97 \\
& Ing & 1.39 & 2.30 \\
\multirow{3}{*}{ Balloon } & lat & 0.77 & 0.85 \\
& vrt & 1.53 & 1.36 \\
& Ing & 3.20 & 3.15 \\
FM & lat & 1.70 & 1.35 \\
& vrt & 0.88 & 0.71 \\
& Ing & 1.16 & 0.46 \\
& lat & 0.46 & 0.37 \\
\hline
\end{tabular}

uncertainties. Furthermore, the IGRT modalities CBCT and $\mathrm{kV} / \mathrm{kV}$ FM imaging were compared with each other in the context of PTV margin determination.

\section{Patients and methods}

Study design and patient selection

From January 2009 to April 2012, 20 non-consecutive patients with histologically proven low- (LR), intermediate(IR) or high-risk (HR) adenocarcinoma of the prostate were treated according to the CHHIP (Conventional or Hypofractionated High Dose Intensity Modulated Radiotherapy for Prostate Cancer) protocol [8], and according to randomization VMAT (RapidArc ${ }^{\circ}$ ) was used to a dose of $74 \mathrm{~Gy} / 37 \mathrm{f}(\mathrm{n}=14), 60 \mathrm{~Gy} / 20 \mathrm{f}(\mathrm{n}=1)$ or $57 \mathrm{~Gy} /$ 19f $(\mathrm{n}=5)$ at the University Hospital Zürich, Switzerland [9]. Hypofractionation schedules were used only for patients randomized within the CHHIP trial (open between 2010 and 2011 at Zurich) and not off-study, since hypofractionation was not standard at that time. Planning CT scans, $\mathrm{kV} / \mathrm{kV}$ images and CBCT's of the 20 patients were retrospectively analysed with ethics approval (Department of Radiation Oncology, University Hospital Zurich). 
Table 2 Systematic $\Sigma$ and random $\sigma$ setup error depending on surrogate used for matching the $\mathrm{CBCT}$ to the reference $\mathrm{CT}$

\begin{tabular}{llll}
\hline & & $\boldsymbol{\Sigma}(\mathbf{m m})$ & $\boldsymbol{\sigma}(\mathbf{m m})$ \\
\hline Bones & vrt & 1.92 & 1.87 \\
& Ing & 1.72 & 1.69 \\
\multirow{3}{*}{ Balloon } & lat & 0.61 & 0.90 \\
& vrt & 1.68 & 1.82 \\
Soft tissue & Ing & 1.20 & 1.66 \\
& vrt & 0.77 & 1.09 \\
& Ing & 0.36 & 0.17 \\
& & 0.49 & 0.93 \\
\hline
\end{tabular}

Fiducial marker matching in CBCT was taken as a reference.

\section{Target definition and treatment planning}

Two weeks prior to CT simulations, 3 FM were placed into the prostate under rectal ultrasound guidance. Non-contrasted planning CT simulation with axial slices thicknesses of $2.0 \mathrm{~mm}$ was performed in supine position by use of a leg holder immobilization device, empty rectum with endorectal balloon (ERB) and full bladder. Reference points were tattooed on the skin.

CTV was defined as prostate (P) only for LR, prostate plus $1 / 3$ of the seminal vesicles (PSV) for IR or the prostate plus the seminal vesicles for HR cancer patients. In order to evaluate the delineation error of prostate only or prostate/ seminal vesicles, the $C_{T V}$ for 5 pts with $L R$ and $\mathrm{CTV}_{\mathrm{PSV}}$ for 5 pts with HR prostate cancer were contoured independently by 3 experienced radiation oncologists using non fused MR images with rectal coil. No formal contouring training was performed.

\section{Treatment}

Daily localization of the prostate position was performed using FM before treatment. CBCT was performed on day
1 to 3 , then once every week (hypofractionation: $6 \times 6$ $\mathrm{CBCT}$, normofractionation: $14 \times 9-10 \mathrm{CBCT}$ ). Additional in-between $\mathrm{CBCT}$ were not used for evaluation. For 10 patients (57Gy/19f $(n=1), 74 G y / 37 f(n=9))$ an additional $\mathrm{CBCT}$ was performed after each fraction to assess intrafractional motion once a week $(1 \times 4 \mathrm{CBCT}, 9 \times 7 \mathrm{CBCT})$. Due to patient's distress, failure of CBCT or correlation function, not all post-treatment $\mathrm{CBCT}$ could be used for evaluation. On day 1 the isocenter after CBCT based corrections was marked on the patient skin. Online isocenter positioning was performed based on FM using $\mathrm{CBCT}$ - as indicated above - or $\mathrm{kV}$ image pairs. $\mathrm{kV} / \mathrm{kV}$ images were performed before each fraction except when a weekly or in-between CBCT was applied. On days with post-treatment $\mathrm{CBCT}$ a $\mathrm{kV} / \mathrm{kV}$ image pair was performed for comparison reason $(14 \times 37 \mathrm{kV} / \mathrm{kV}+5 \times$ $19 \mathrm{kV} / \mathrm{kV}+1 \times 20 \mathrm{kV} / \mathrm{kV}=633 \mathrm{kV} / \mathrm{kV}-172 \mathrm{kV} / \mathrm{kV}$ (pre-CBCT days) $+67 \mathrm{kV} / \mathrm{kV}$ (post-CBCT days) $=528 \mathrm{kV} /$ $\mathrm{kV}-21 \mathrm{kV} / \mathrm{kV}$ (in-between CBCT) $=507 \mathrm{kV} / \mathrm{kV})$.

\section{Statistical analysis}

The van Herk formula $\mathrm{M}=2.5 \Sigma+0.7 \sigma$ was used for PTV margin calculation, where $\Sigma$ represents the systematic and $\sigma$ the random uncertainty. Random uncertainties are statistical fluctuations whereas systematic uncertainties are often due to a problem which persists throughout the entire treatment. We calculated systematic uncertainty for intra-fractional motion, contouring as well as setup. Random uncertainties were calculated for intra-fractional motion and patient setup. Total $\Sigma$ was then calculated: $\Sigma=\left(\sum_{\text {contour }}^{2}+\Sigma_{\text {patient setup }}^{2}+\sum_{\text {intrafraction motion }}^{2}\right)^{1 / 2}$ as well as total $\sigma: \sigma=\left(\sigma_{\text {patient setup }}^{2}+\sigma_{\text {intrafraction motion }}^{2}\right)^{1 / 2}$. Statistical analysis was performed with MATLAB, version 11 (Mathworks, MA, U.S.A.). We used a $\sigma_{\mathrm{p}}-$ where $\sigma_{\mathrm{p}}$ is the standard deviation of the normal dose penumbra - of $3 \mathrm{~mm}$ assuming the worst case (cranial-caudal direction)

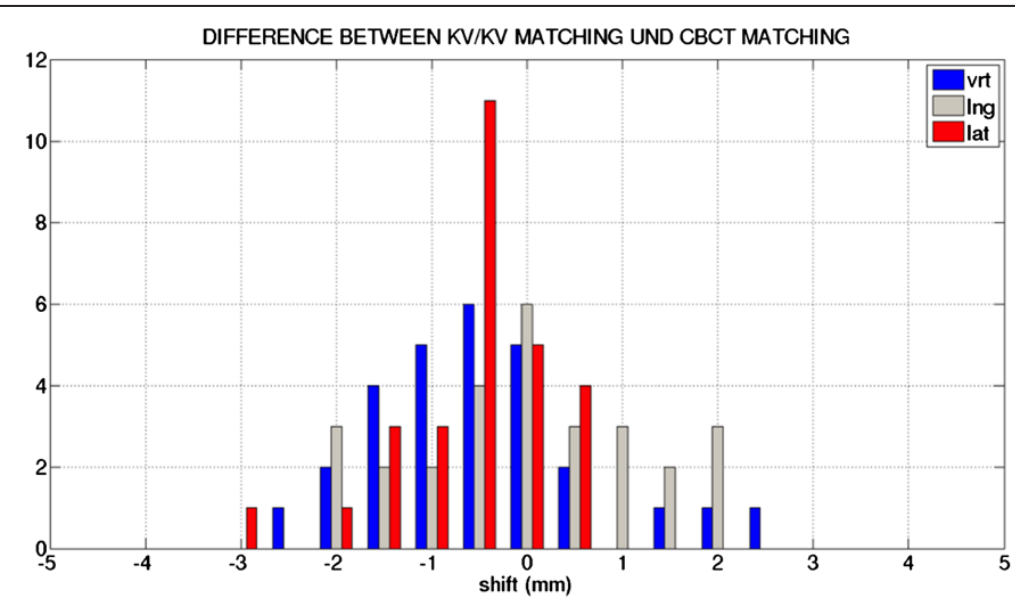

Figure 2 Bar diagram of differences in vertical (vrt), longitudinal (lng) and lateral (lat) direction between CBCT and $\mathrm{kV} / \mathrm{kV}$ setup matching on fiducial markers $(\mathrm{N}=10)$. 


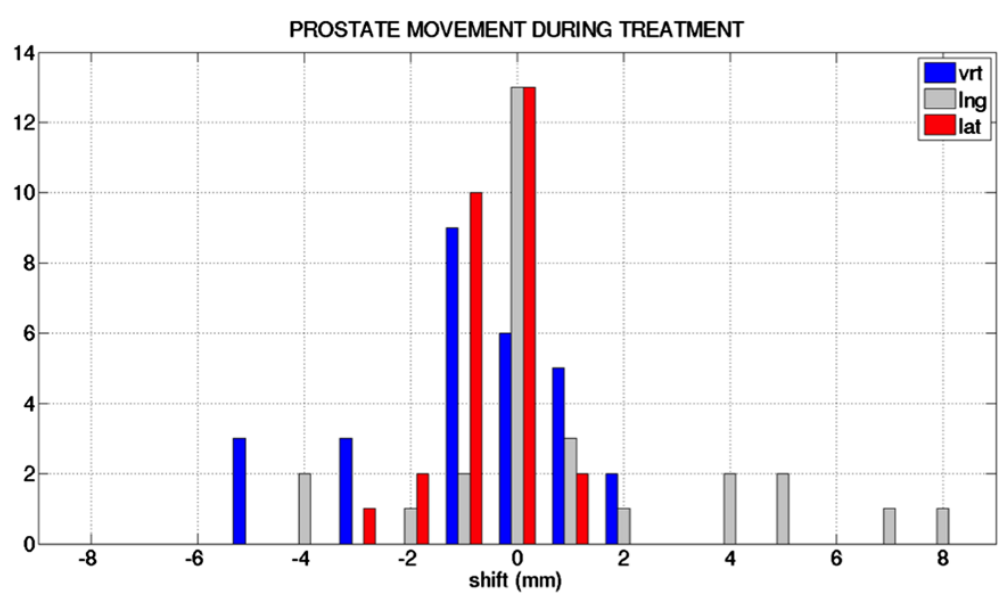

Figure 3 Bar diagram of intra-fraction motion in vertical (vrt), longitudinal (lng) and lateral (lat) direction during treatment ( $\mathrm{N}=10)$. Intra-fraction motion was determined by fiducial marker difference between pre- and post- CBCT.

because for VMAT $\sigma_{\mathrm{p}}$ is larger in the left/right as well as anterior/posterior direction compared to the cranial/caudal direction. Furthermore, changing the $\sigma_{\mathrm{p}}$ influences only the random error and results in a small change of the margin.

The online match was based on FM. Additionally offline matches based on bony anatomy and ERB $(\mathrm{kV} / \mathrm{kV}$ image pairs and $\mathrm{CBCT}$ ), as well as soft prostate tissue (CBCT) were performed to compare different matching techniques. The setup uncertainty was calculated for each of the different matching techniques in respect to the online performed FM match. To assess the difference between FM match in $\mathrm{kV}$ image pairs and $\mathrm{CBCT}$ the two imaging modalities were performed strictly consecutively and shifts were compared. To assess intra-fraction prostate motion, differences between FM position in pre-treatment and post-treatment $\mathrm{CBCTs}$ were established.

The contouring uncertainties of the $\mathrm{CTV}_{\mathrm{P}}$ and the $\mathrm{CTV}_{\mathrm{PSV}}$ were determined by comparing intersection and union of 2 corresponding contours using a selfdeveloped analysis script in Matlab (Mathworks): The prostate volume was divided into 6 subsections (anterior, posterior, left, right, cranial and caudal) (Figure 1). For each subsection the mean distance between the union and

Table 3 Average contouring errors for prostate (P) or prostate with seminal vesicles (PSV) in anterior (vrt/ anterior), posterior (vrt/posterior), longitudinal (long) and lateral (lat) direction

\begin{tabular}{lll}
\hline & $\mathbf{P}(\mathbf{m m})$ & PSV $(\mathbf{m m})$ \\
\hline vrt & 1.52 & 2.57 \\
vrt/anterior & 1.98 & 3.49 \\
vrt/posterior & 1.04 & 1.58 \\
Long & 2.03 & 3.20 \\
Lat & 1.68 & 2.52 \\
\hline
\end{tabular}

the intersection was calculated. The mean and standard deviation (SD) between the pairwise comparisons were calculated. Wilcoxon-Mann-Whitney-Test was used to compare contouring uncertainties for P and PSV.

\section{Results}

Setup (inter-fractional) uncertainty

172 and 52 CBCT before and after RT and $507 \mathrm{kV} / \mathrm{kV}$ images before RT were analyzed. The setup uncertainty was calculated for the matching on different surrogates in $\mathrm{kV}$ images pairs as well as CBCT using CBCT FM match as reference. The mean systematic and random setup uncertainties for each modality are shown in Tables 1 and 2. The difference in setup using FM between $\mathrm{CBCT}$ and $\mathrm{kV} / \mathrm{kV}$ was usually below $2 \mathrm{~mm}$ (Figure 2). Matching on $\mathrm{FM}$ in $\mathrm{kV} / \mathrm{kV}$ images or matching on soft tissue in CBCT lead to systematic and random uncertainties below $1 \mathrm{~mm}$. Bony anatomy match as well as balloon match increased the systematic as well as the random uncertainty. This was more pronounced if $\mathrm{kV} / \mathrm{kV}$ image pairs were used compared to the use of CBCT. Large systematic as well as random uncertainties in longitudinal direction were found with the use of the ERB as a surrogate for prostate position since reproducible placing of the ERB was not possible.

\section{Intra-fractional motion uncertainty}

For intra-fractional motion uncertainty calculation of the prostate FM shift of pre- and post-treatment CBCT's were compared with each other (time between 3 to $6 \mathrm{~min})$. Maximum FM shifts reached $5 \mathrm{~mm}$ in vertical and $8 \mathrm{~mm}$ in longitudinal direction (Figure 3). The systematic intra-fractional uncertainty was $1.39 \mathrm{~mm}$ (vrt), $1.36 \mathrm{~mm}$ (lng), $0.92 \mathrm{~mm}$ (lat) and the day to day random uncertainty was $1.62 \mathrm{~mm}$ (vrt), $1.39 \mathrm{~mm}$ (lng), $0.97 \mathrm{~mm}$ (lat), respectively. 
Table 4 Margins needed to be applied for patients with low risk prostate cancer when matching on fiducial markers (FM), soft tissue (ST), bones (B) or balloon (ERB)

\begin{tabular}{llllllll}
\hline & $\mathbf{M}(\mathbf{m m}) \mathbf{C B C T}$ & $\mathbf{S T}(\mathbf{m m}) \mathbf{C B C T}$ & $\mathbf{B}(\mathbf{m m}) \mathbf{C B C T}$ & $\mathbf{B a}(\mathbf{m m}) \mathbf{C B C T}$ & $\mathbf{M}(\mathbf{m m}) \mathbf{k V}$ & $\mathbf{B}(\mathbf{m m}) \mathbf{k V}$ & $\mathbf{B a}(\mathbf{m m}) \mathbf{k V}$ \\
\hline Anterior & 7.18 & 7.69 & 9.45 & 9.07 & 7.67 & 8.64 & 8.64 \\
Posterior & 5.47 & 6.09 & 8.20 & 7.75 & 6.10 & 7.27 & 7.27 \\
Long & 7.09 & 7.16 & 9.00 & 8.32 & 7.79 & 12.48 & 12.48 \\
Lat & 5.47 & 5.88 & 5.95 & 6.10 & 5.65 & 7.58 & 7.58 \\
\hline
\end{tabular}

\section{Contouring error}

Systematic contouring uncertainties were larger for PSV than for $\mathrm{P}(p=0.012)$. The mean systematic uncertainty was largest in the longitudinal axis as shown in Table 3. In vertical direction the uncertainty was found to be larger in anterior $(1.98 \mathrm{~mm}(\mathrm{P}), 3.49 \mathrm{~mm}$ (PSV)) than posterior $(1.04 \mathrm{~mm}(\mathrm{P}), 1.58 \mathrm{~mm}(\mathrm{PSV})$ direction.

\section{PTV margin (according to van Herk formula)}

The calculated PTV margins can be found in Tables 4 and 5. The margin for P only was calculated to be $7.2 \mathrm{~mm}$ in anterior, $5.5 \mathrm{~mm}$ posterior, $7.1 \mathrm{~mm}$ longitudinal, and $5.5 \mathrm{~mm}$ in lateral direction. PTV margins were larger for PSV volumes than for P only volumes due to the contouring differences. The difference was largest in longitudinal direction $(2.5 \mathrm{~mm})$. The contouring uncertainty contributed most to the PTV margin compared with the intra-fractional and setup uncertainties. Matching on soft tissue in the $\mathrm{CBCT}$ or on $\mathrm{FM}$ in $\mathrm{kV} / \mathrm{kV}$ image pairs increased the margin by less than $0.7 \mathrm{~mm}$. Matching on the ERB required an additional margin of up to $5.4 \mathrm{~mm}$.

\section{Discussion}

In the IMRT-IGRT-era, PTV margin determination has become of crucial importance $[3,10]$. We found a PTV margin between 5-8 $\mathrm{mm}$ for the $\mathrm{P}$ (LR) and between 6-11 $\mathrm{mm}$ for the PSV (IR/HR). A similar prostate PTV margin of $7 \mathrm{~mm}$ was determined by van Herk and colleagues though intra-fractional motion was not considered in their calculation and no ERB was used [11]. PTV margins used at the institution based on the CHHIP protocol and were $1 \mathrm{~cm}$ in all directions for PTV1 (PSV), $1 \mathrm{~cm} / 5 \mathrm{~mm}$ (posterior) for PTV2 (P) and $5 \mathrm{~mm} / 0 \mathrm{~mm}$ (posterior) for PTV3 (P) managing the risk of rectal toxicity [8]. However use of IGRT was not required to compensate for organ motion. We found that random errors produced by intra-fraction (organ motion) and inter-fraction (setup) uncertainties were of similar magnitude. Interestingly, the largest uncertainty was introduced by CTV delineation (LR PC: 1 - 2 mm, IR/HR PC: 1.6 - $3.5 \mathrm{~mm}$ ). Patient positioning like bone or ERB matching resulted in considerably larger PTV margins [12]. Assuming an optimal delineation error of $1 \mathrm{~mm}$ our recommendations for an anisotropic PTV margin are as follows: $\mathrm{PTV}_{\mathrm{P}} 6.2 \mathrm{~mm}$ (ant), $5.43 \mathrm{~mm}$ (post), $6.06 \mathrm{~mm}$ (lng) and $4.79 \mathrm{~mm}$ (lat), PTV PSV $8.03 \mathrm{~mm}$ (ant), $5.81 \mathrm{~mm}$ (post), $7.47 \mathrm{~mm}$ (lng) and $6.87 \mathrm{~mm}$ (lat). Application of organ tracking would allow for further PTV lowering.

Image-guided patient positioning considerably reduced PTV margin by about 2- $4.7 \mathrm{~mm}$ compared with bone or ERB matching which is known from the literature [10]. IGRT modalities such as CBCT and $\mathrm{kV} / \mathrm{kV}$-image pairs with FM or CBCT soft tissue matching were interchangeable in respect of accuracy. While some studies have shown the same, others have found that identification of FM on volumetric or planar images was not equivalent $( \pm 3 \mathrm{~mm})$ [5]. However, one needs to keep in mind intra-fractional prostate motion between imaging modalities.

In this study, the intra-fractional prostate movement was generally below $2 \mathrm{~mm}$ ( $\Sigma$ was $0.36-0.75 \mathrm{~mm}$ ) for treatment duration of around 90 seconds, similar to results from the literature $(\Sigma 1-2 \mathrm{~mm})$ [13]. Prolonged RT duration can increase the intra-fractional prostate movement up to 3-6 $\mathrm{mm}$ [14]. Using an ERB might decrease intra-fractional prostate motion. Importantly, the SV move independently from the prostate gland with an uncertainty for PTV between $1.4-4.5 \mathrm{~mm}$ and should be considered in future margin protocols [15].

Table 5 Margins needed to be applied for patients with high risk prostate cancer when matching on fiducial markers (FM), soft tissue (ST), bones (B) or balloon (ERB)

\begin{tabular}{llllllll}
\hline & $\mathbf{M}(\mathbf{m m}) \mathbf{C B C T}$ & $\mathbf{S T}(\mathbf{m m}) \mathbf{C B C T}$ & $\mathbf{B}(\mathbf{m m}) \mathbf{C B C T}$ & $\mathbf{B a}(\mathbf{m m}) \mathbf{C B C T}$ & $\mathbf{M}(\mathbf{m m}) \mathbf{k V}$ & $\mathbf{B}(\mathbf{m m}) \mathbf{k V}$ & $\mathbf{B a}(\mathbf{m m}) \mathbf{k V}$ \\
\hline Anterior & 10.52 & 10.94 & 12.28 & 11.99 & 10.88 & 12.18 & 11.62 \\
Posterior & 6.39 & 6.94 & 8.85 & 8.44 & 6.94 & 8.68 & 7.99 \\
Long & 9.67 & 9.72 & 11.23 & 10.71 & 10.19 & 11.24 & 14.23 \\
Lat & 7.39 & 7.76 & 7.80 & 7.95 & 7.53 & 7.88 & 9.11 \\
\hline
\end{tabular}

For low risk prostate cancer patients the prostate only was treated. For high risk prostate cancer patients the prostate and the seminal vesicles were treated. 
Remarkably, the largest uncertainties for PTV margins were produced by the radiation oncologist's CTV delineation, particularly in the longitudinal axis. In the literature, the inter-observer variation for contouring has been reported to be largest in regions near the seminal vesicles and the apex [16]. Several geometrical uncertainties are involved in the delineation process including imaging resolution [6]. CTV delineation variation can be significantly reduced using MRI, teaching/protocols or automated model-based organ segmentation [17].

In practice, for protocols such as RTOG 0938 trial, a randomized phase II trial on hypofractionated radiation therapy, PTV was defined as the CTV $\mathrm{p}_{\mathrm{p}}$ plus $5 \mathrm{~mm}$ in all dimensions except $3 \mathrm{~mm}$ posteriorly or anteriorly if necessary [18]. However, reducing treatment margins based on improved treatment accuracy may lead to geographical miss and serious underdosage of the CTV [7]. In order to prevent a geographical miss adaptive IGRT (-2.5-4.5 $\mathrm{mm})$ might be recommended with daily imaging, especially in hypofractionation protocols [19].

The preference of IGRT modality is subject to various considerations. In our study we have chosen FM match in $\mathrm{CBCT}$ as reference since the stability of implanted markers was shown by several studies with an average seed migration of $1.2+/-0.2 \mathrm{~mm}$ (seeds) and $0.8 \mathrm{~mm}+/-0.6 \mathrm{~mm}$ (coils) [20]. Advantages of $\mathrm{kV} / \mathrm{kV}$ image pairs compared to CBCT are reduced acquisition/matching time, imaging dose and lower cost. However, it is an invasive procedure with the risk for prostate infection. In our opinion, FM and CBCT complement each other, especially in view of adaptive and real-time tumor tracking [21].

\section{Consent}

Written informed consent was obtained from the patient for data evaluation and publication; in case of participation in the CHIPP trial an additional informed consent was obtained.

\section{Competing interests}

On behalf of all authors, the corresponding author states that there are no conflicts of interest.

\section{Authors' contributions}

Concept and design: ZD, OC, LS, DP. Study material and patients: TA, NY, SG, ZD, GC. Collection and assembly of data: DP, OC, ZD, LS, BC, TA. Data analysis and interpretation: LS, OC, ZD, KS. Manuscript writing: OC, ZD, LS. All authors read and approved the final manuscript.

Received: 28 January 2014 Accepted: 3 October 2014

Published online: 11 November 2014

\section{References}

1. Zelefsky MJ, Kollmeier M, Cox B, Fidaleo A, Sperling D, Pei X, Carver B, Coleman J, Lovelock M, Hunt M: Improved clinical outcomes with high-dose image guided radiotherapy compared with non-IGRT for the treatment of clinically localized prostate cancer. Int I Radiat Oncol Biol Phys 2012, 84:125-129.
2. Dearnaley DP, Sydes MR, Graham JD, Aird EG, Bottomley D, Cowan RA, Huddart RA, Jose CC, Matthews JH, Millar J, Moore AR, Morgan RC, Russell JM, Scrase CD, Stephens RJ, Syndikus I, Parmar MK, RT01 collaborators: Escalated-dose versus standard-dose conformal radiotherapy in prostate cancer: first results from the MRC RT01 randomised controlled trial. Lancet Oncol 2007, 8:475-487.

3. Crehange G, Mirjolet C, Gauthier M, Martin E, Truc G, Peignaux-Casasnovas K, Azelie C, Bonnetain F, Naudy S, Maingon P: Clinical impact of margin reduction on late toxicity and short-term biochemical control for patients treated with daily on-line image guided IMRT for prostate cancer. Radiotherapy and oncology: journal of the European Society for Therapeutic Radiology and Oncology 2012, 103:244-246.

4. Langsenlehner T, Doller C, Winkler P, Galle G, Kapp KS: Impact of inter- and intrafraction deviations and residual set-up errors on PTV margins. Different alignment techniques in 3D conformal prostate cancer radiotherapy. Strahlentherapie und Onkologie. Organ der Deutschen Rontgengesellschaft [et al] 2013, 189:321-328.

5. Barney BM, Lee RJ, Handrahan D, Welsh KT, Cook JT, Sause WT: Image-guided radiotherapy (IGRT) for prostate cancer comparing $\mathrm{kV}$ imaging of fiducial markers with cone beam computed tomography (CBCT). Int J Radiat Oncol Biol Phys 2011, 80:301-305.

6. Rasch C, Steenbakkers $\mathrm{R}$, van Herk M: Target definition in prostate, head, and neck. Semin Radiat Oncol 2005, 15:136-145.

7. van Herk M, Remeijer P, Rasch C, Lebesque JV: The probability of correct target dosage: dose-population histograms for deriving treatment margins in radiotherapy. Int J Radiat Oncol Biol Phys 2000, 47:1121-1135.

8. Dearnaley D: CHHiP: Conventional or hypofractionated high dose intensity modulated radiotherapy for prostate cancer, protocol version 8.0. Sutton, Surrey, UK: Institute of Cancer Research. Clinical Trial \& Statistics Unit 2009, 52

9. Zwahlen DR, Lang S, Hrbacek J, Glanzmann C, Kloeck S, Najafi Y, Streller T, Studer G, Zaugg K, Luetolf UM: The use of photon beams of a flattening filter-free linear accelerator for hypofractionated volumetric modulated arc therapy in localized prostate cancer. Int J Radiat Oncol Biol Phys 2012 83:1655-1660.

10. Skarsgard D, Cadman P, El-Gayed A, Pearcey R, Tai P, Pervez N, Wu J: Planning target volume margins for prostate radiotherapy using daily electronic portal imaging and implanted fiducial markers. Radiat Oncol 2010, 5:52.

11. van Herk M: Errors and margins in radiotherapy. Semin Radiat Oncol 2004, 14:52-64.

12. Nairz O, Merz F, Deutschmann H, Kopp P, Schöller H, Zehentmayr F, Wurstbauer K, Kametriser G, Sedlmayer F: A strategy for the use of image-guided radiotherapy (IGRT) on linear accelerators and its impact on treatment margins for prostate cancer patients. Strahlentherapie und Onkologie. Organ der Deutschen Rontgengesellschaft [et al] 2008, 184:663-667.

13. Adamson J, Wu Q, Yan D: Dosimetric effect of intrafraction motion and residual setup error for hypofractionated prostate intensity-modulated radiotherapy with online cone beam computed tomography image guidance. Int J Radiat Oncol Biol Phys 2011, 80:453-461.

14. Polat B, Guenther I, Wilbert J, Goebel J, Sweeney RA, Flentje M, Guckenberger $M$ : Intra-fractional uncertainties in image-guided intensity-modulated radiotherapy (IMRT) of prostate cancer. Strahlentherapie und Onkologie. Organ der Deutschen Rontgengesellschaft [et al] 2008, 184:668-673.

15. Mak D, Gill S, Paul R, Stillie A, Haworth A, Kron T, Cramb J, Knight K, Thomas J, Duchesne $G$, Foroudi F: Seminal vesicle interfraction displacement and margins in image guided radiotherapy for prostate cancer. Radiat Oncol 2012, 7:139.

16. Remeijer P, Rasch C, Lebesque JV, van Herk M: A general methodology for three-dimensional analysis of variation in target volume delineation. Med Phys 1999, 26:931-940.

17. Khoo EL, Schick K, Plank AW, Poulsen M, Wong WW, Middleton M, Martin JM: Prostate contouring variation: can it be fixed? International Journal of Radiation Oncology, Biology. Physics 2012, 82:1923-1929.

18. Lukka H: A randomized pphase II trial of hypofractionated radiotherapy for favorable-risk prostate cancer - RTOG CCOP study; 2013.

19. Deutschmann $H$, Kametriser $G$, Steininger $P$, Scherer $P$, Schöller $H$, Gaisberger C, Mooslechner M, Mitterlechner B, Weichenberger H, Fastner G, Wurstbauer K, Jeschke S, Forstner R, Sedlmayer F: First clinical release of an online, adaptive, aperture-based image-guided radiotherapy strategy in intensity-modulated radiotherapy to correct for inter- and intrafractional rotations of the prostate. Int J Radiat Oncol Biol Phys 2012, 83:1624-1632. 
20. Gates LL, Gladstone DJ, Kasibhatla MS, Marshall JF, Seigne JD, Hug E, Hartford AC: Stability of serrated gold coil markers in prostate localization. Journal of Applied Clinical Medical Physics/American College of Medical Physics 2011, 12:3453.

21. Piziorska M, Kukolowicz P, Zawadzka A, Pilichowska M, Peczkowski P: Adaptive off-line protocol for prostate external radiotherapy with cone beam computer tomography. Strahlentherapie und Onkologie. Organ der Deutschen Rontgengesellschaft [et al] 2012, 188:1003-1009.

doi:10.1186/s13014-014-0229-z

Cite this article as: Oehler et al:: PTV margin definition in

hypofractionated IGRT of localized prostate cancer using cone beam CT and orthogonal image pairs with fiducial markers. Radiation Oncology 2014 9:229.

\section{Submit your next manuscript to BioMed Central and take full advantage of:}

- Convenient online submission

- Thorough peer review

- No space constraints or color figure charges

- Immediate publication on acceptance

- Inclusion in PubMed, CAS, Scopus and Google Scholar

- Research which is freely available for redistribution 